\title{
Extracorporeal life support for adults with refractory septic shock
}

Sun Kyun Ro, MD, PhD, ${ }^{\text {a,b }}$ Wan Kee Kim, MD, ${ }^{\mathrm{b}}$ Ju Yong Lim, MD, ${ }^{\mathrm{b}}$ Jae Suk Yoo, MD, ${ }^{\mathrm{c}}$ Sang-Bum Hong, MD, PhD, ${ }^{\mathrm{d}}$ and Joon Bum Kim, MD, $\mathrm{PhD}^{\mathrm{b}}$

\section{ABSTRACT}

Objective: Although the use of extracorporeal membrane oxygenation (ECMO) in shock patients is increasing worldwide, studies concerning this treatment for adult septic shock are limited. This study aimed to analyze the outcome of venoarterial ECMO in adult patients with septic shock refractory to conventional treatment.

Methods: A total of 71 consecutive patients who presented with septic shock and underwent venoarterial ECMO were reviewed. Clinical parameters were compared between survivors and nonsurvivors. Weaning and survival outcomes of these patients were compared with the control group of 253 patients who received venoarterial ECMO for cardiogenic shock.

Results: The mean age was $56.0 \pm 12.3$ years. Of the 71 septic shock patients, 11 $(15.5 \%)$ were successfully weaned from ECMO after a median of 7.9 [interquartile range (IQR), 6.3-10.2] days, 5 of whom (7.0\%) survived to discharge. Pre- and 6 hours post-procedural lactate levels were significantly higher in the nonsurvivors (11.6 [IQR, 7.5-15.0] vs 5.8 [IQR, 4.3-5.9], $P=.036 ; 15.0$ [IQR, 11.1-15.0] vs 5.2 [IQR, 4.7-5.4], $P=.002$ ). Rates of successful weaning from venoarterial ECMO $(15.5 \%$ vs $45.5 \%)$, and of survival up to hospital discharge $(7.0 \%$ vs $28.9 \%)$ were significantly lower in septic shock than in cardiogenic shock patients $(\mathrm{n}=253 ; P<.001)$.

Conclusions: Outcomes of ECMO in refractory septic shock patients were poor with a very low probability of survival. This finding raises questions concerning the utility of applying ECMO for medically refractory septic shock. Elevated arterial lactate levels pre- and post-ECMO were associated with risk of in-hospital death. Further large-scale studies are needed to validate the results of this study. (J Thorac Cardiovasc Surg 2018;156:1104-9)

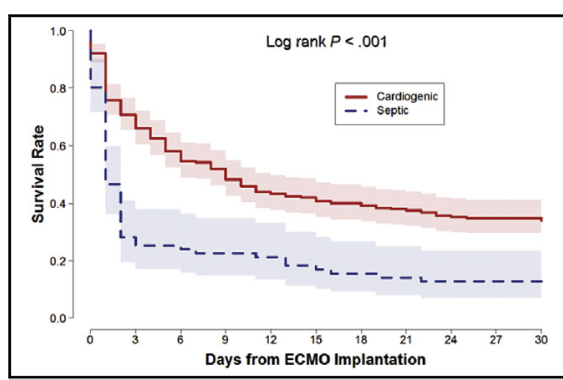

Overall survival of the septic shock and the cardiogenic shock groups.

\section{Central Message}

The outcomes of ECMO in refractory septic shock were poor with very low survival probabilities. This finding raises a futility issue of applying ECMO for medically refractory septic shock.

\section{Perspective}

Despite the increasing use of extracorporeal life support, studies of venoarterial ECMO for adult septic shock are limited. Because of the poor survival outcomes for venoarterial ECMO in septic shock patients compared with nonseptic shock patients in this study, the benefit of venoarterial ECMO in adult septic shock patients remains uncertain.

See Editorial Commentaries pages 1110 and 1112.
Since extracorporeal life support (ECLS) was first introduced as a salvage treatment for cardiac arrest unresponsive

\footnotetext{
From the a Department of Thoracic and Cardiovascular Surgery, Hanyang University Guri Hospital, Hanyang University College of Medicine, Seoul, South Korea; Departments of ${ }^{\mathrm{b}}$ Thoracic and Cardiovascular Surgery and ${ }^{\mathrm{d}}$ Pulmonary and Critical Care Medicine, Asan Medical Center, University of Ulsan College of Medicine, Seoul, South Korea; and ${ }^{\mathrm{c}}$ Department of Thoracic and Cardiovascular Surgery, Sejong Hospital, Buchoen, South Korea.

Received for publication Sept 27, 2017; revisions received March 2, 2018; accepted for publication March 24, 2018; available ahead of print May 9, 2018.

Address for reprints: Joon Bum Kim, MD, PhD, Department of Thoracic and Cardiovascular Surgery, Asan Medical Center, University of Ulsan College of Medicine, 88, Olympic-ro 43-gil, Songpa-gu, Seoul 138-736, South Korea (E-mail: jbkim1975@amc.seoul.kr).

$0022-5223 / \$ 36.00$

Copyright (c) 2018 by The American Association for Thoracic Surgery

https://doi.org/10.1016/j.jtcvs.2018.03.123
}

to conventional treatment in the $1960 \mathrm{~s},{ }^{1}$ miniaturization of membrane oxygenators and the centrifugal pumps has made the heart-lung machine compact and portable, and thinwalled cannulae have enabled rapid implantation of the device via peripheral routes. ${ }^{2}$ These technical developments have led to widespread use of ECLS. The application of venoarterial extracorporeal membrane oxygenation (ECMO) has also been expanded to a variety of conditions such as

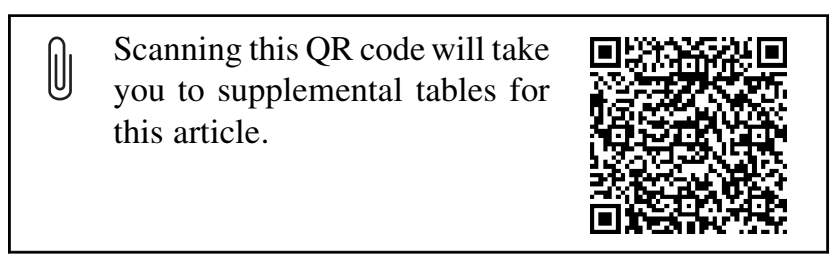




\section{Abbreviations and Acronyms \\ ECLS = extracorporeal life support \\ $\mathrm{ECMO}=$ extracorporeal membrane oxygenation \\ $\mathrm{IQR}=$ interquartile range \\ SOFA $=$ sepsis-related organ failure assessment}

septic shock unresponsive to conventional treatment, rather than being confined to cardiogenic shock, the traditional indication for venoarterial ECMO. However, the role of venoarterial ECMO in septic shock remains controversial and the clinical outcomes were inconsistent. Whereas some authors reported in-hospital mortalities of $85 \%$ (44 of 52) and $78 \%$ ( 25 of 32 ), others reported a satisfactory in-hospital mortality of $29 \%$ (4 of 14 ). ${ }^{3-5}$ Moreover, publications concerning the clinical outcomes of venoarterial ECMO in adult patients with refractory septic shock are limited. We aimed to analyze the early outcomes of venoarterial ECMO performed in patients presenting with refractory septic shock and to identify risk factors for post-ECMO mortality.

\section{METHODS}

\section{Study Populations}

All patients who underwent ECMO at our institution were prospectively registered in the ECMO database, which consists of baseline patient characteristics, detailed information on the ECMO setup, periprocedural complications, and in-hospital outcomes. We reviewed this registry to identify adult patients (age 20 years or older) who underwent venoarterial ECMO support to manage refractory shocks of various origins from January 2005 through August 2012. Patients who initially received venovenous ECMO were not included in the study cohort. Of 339 patients identified who met inclusion criteria, 71 patients $(20.9 \%)$ received ECMO for septic shock and they were selected for the main study cohort. Of the remaining 268 patients $(79.1 \%), 253$ patients who received ECMO for cardiogenic shock $(74.6 \%)$ served as the comparator group, with the exclusion of 15 patients $(4.4 \%)$, who received ECMO for hypovolemic shock or other causes to maintain the homogeneity of the group.

The study was approved by the institutional ethics committee/review board of the Asan Medical Center, Seoul, Korea, and the requirement for informed patient consent was waived in view of the retrospective nature of the study.

\section{Indication for ECMO and Management}

Severe sepsis was defined as acute organ dysfunction secondary to infection and septic shock was defined as severe sepsis with hypotension not reversed by fluid resuscitation. When the patients were diagnosed as septic shock, they were primarily treated with aggressive fluid challenge, adequate antibiotics, and vasoactive agents. ${ }^{6}$ Venoarterial ECMO was indicated only for patients with persistent circulatory collapse despite these aggressive medical treatments. When the attending physician called the ECMO team, the on-call cardiovascular surgeon of the ECMO team decided whether to deploy ECMO and, if so, the cardiovascular surgeon in charge managed the patient thereafter.

Peripheral cannulation was performed in all patients; the common femoral artery was used as an arterial cannulation route in all patients and the common femoral vein, internal jugular vein, and both were used as the venous cannulation route in $65(91.7 \%), 2(2.8 \%)$, and 4 patients
$(5.6 \%)$, respectively. In most cases arterial and venous cannulae could be implanted percutaneously using the Seldinger technique, but cutdown procedures were unavoidable in a few cases. Either 14- to 21-French arterial cannulae or 17- to 28 -French venous drainage cannulae were used depending on the individual patient's body surface area. The ECMO system consisted of a centrifugal pump, a hollow-fiber membrane oxygenator with an integral heat exchanger, and a heparin-coated circuit. Three types of ECMO system were used; the Capiox Emergency Bypass System (Terumo, Tokyo, Japan), the Permanent Life Support system (MAQUET, Hirrlingen, Germany), and the Bio-Consol 560 system (Medtronic, Minneapolis, Minn).

Administration of an intravenous heparin bolus of 50 to $100 \mathrm{U} / \mathrm{kg}$ before cannulation was followed by continuous intravenous heparin infusion to maintain the activated clotting time within 180 to 200 seconds. In patients with a bleeding tendency, nafamostat mesilate (Futhan; Torii Pharmaceutical, Tokyo, Japan) was used as an anticoagulant alternative to heparin depending on the physician's judgement. ${ }^{7,8}$ An antegrade perfusion catheter for distal limb perfusion was routinely inserted distal to the arterial cannulation site, and the flow through the dorsalis pedis and posterior tibial arteries of the affected limb was assessed using a portable Doppler device. The arterial cannula was repositioned to the opposite side of the femoral artery if the affected limb showed signs of ischemic injury. ECMO blood flow was aimed at a cardiac index of $2.4 \mathrm{~L} / \mathrm{min} / \mathrm{m}^{2}$ or more. Vasoconstrictors such as vasopressin were preferred over inotropic agents for maintaining mean arterial pressure at 60 to $70 \mathrm{~mm} \mathrm{Hg}$, and blood products were transfused appropriately to maintain hematocrit at $30 \%$ to $35 \%$ and platelet count $\geq 100,000 / \mathrm{mm}^{3}$.

Several parameters were used to determine the timing of weaning from ECMO, including increased mean arterial pressure, increased pulse pressure, and decreased central venous and/or pulmonary artery pressures. Weaning was initiated when echocardiographic findings showed adequate ventricular filling and an ejection fraction $\geq 30 \%$ to $35 \%$ at ECMO flow with a cardiac index of $1.0 \mathrm{~L} / \mathrm{min} / \mathrm{m}^{2}$. Several hemodynamic parameters were assessed while ECMO flow was gradually reduced. If the patient remained stable at an ECMO flow of 0.5 per minute over 30 minutes, ECMO was removed at the bedside. Successful weaning was defined as weaning from ECMO followed by survival for at least 48 hours.?

\section{Data Collection and Statistical Analysis}

In addition to the data available from the ECMO database, the following information was retrieved using a retrospective medical chart review; sepsis-related organ failure assessment (SOFA) score at the start of ECMO implantation, and data regarding pathogenic microorganisms and antibiotic use. Blood laboratory findings (complete blood cell count, blood chemistry, arterial blood gas analysis, blood lactate, etc) just before the initiation of ECLS were also obtained.

Categorical variables are presented as numbers and percentages and were compared using the $\chi^{2}$ and Fisher exact tests. Continuous variables are expressed as mean $\pm \mathrm{SD}$ or median value with interquartile range (IQR) on the basis of the results of the Shapiro-Wilk normality test and were compared using the $t$ test or the Mann-Whitney $U$ test, as appropriate. Survival curves were plotted using the Kaplan-Meier method and survival rates were compared using the log-rank test. $P$ values $<.05$ were considered statistically significant. SPSS version 18.0 (SPSS, Inc, Chicago, Ill) and R statistics, version 3.3.3 (http://www.r-project.org) were used for all statistical analyses.

\section{RESULTS}

\section{Baseline Characteristics}

Mean age was $56.0 \pm 12.3$ years. Table 1 shows a summary of the baseline characteristics, including SOFA scores and circulatory and laboratory parameters of the survivors and nonsurvivors. The mean SOFA score was slightly lower in the survivor group than in the nonsurvivor group, but the 
TABLE 1. Baseline characteristics between survivors and nonsurvivors for patients receiving ECLS for septic shock

\begin{tabular}{|c|c|c|c|}
\hline Variable & Survivors $(n=5)$ & Nonsurvivors $(\mathrm{n}=66)$ & $P$ value \\
\hline Age, y & $58.0(58.0-63.0)$ & $57.5(48.0-65.0)$ & .37 \\
\hline Male sex, n (\%) & $3(60.0)$ & $37(56.1)$ & $>.99$ \\
\hline Body mass index, $\mathrm{kg} / \mathrm{m}^{2}$ & $21.4(19.8-24.7)$ & $22.4(19.5-26.0)$ & .75 \\
\hline Body surface area, $\mathrm{m}^{2}$ & $1.54(1.53-1.64)$ & $1.62(1.51-1.73)$ & .75 \\
\hline Diabetes mellitus, n (\%) & $3(60.0)$ & $16(24.2)$ & .22 \\
\hline Hypertension, n (\%) & $3(60.0)$ & $17(25.8)$ & .26 \\
\hline Chronic renal failure, n (\%) & $0(0)$ & $6(9.1)$ & $>.99$ \\
\hline Liver cirrhosis, n (\%) & $3(60.0)$ & $34(51.5)$ & $>.99$ \\
\hline ARDS, n (\%) & $3(60.0)$ & $31(47.0)$ & .92 \\
\hline Immunosuppressed, n (\%) & $2(40.0)$ & $34(51.5)$ & .67 \\
\hline SOFA score & $18.0(13.0-20.0)$ & $19.0(15.0-22.0)$ & .39 \\
\hline Shock to ECMO time, hours & $4.0(3.7-4.2)$ & $18.0(6.7-53.7)$ & .052 \\
\hline \multicolumn{4}{|l|}{ Circulatory } \\
\hline MAP, mm Hg & $64.3 \pm 10.3$ & $58.3 \pm 11.6$ & .27 \\
\hline Urine output, $\mathrm{mL} / \mathrm{d}$ & $125.0(120.0-875.0)$ & $124.5(25.0-727.0)$ & .61 \\
\hline Use of vasopressin, $n(\%)$ & $1(20.0)$ & $39(59.1)$ & .22 \\
\hline CPR before ECLS, n (\%) & 0 & 9 (13.6) & .85 \\
\hline \multicolumn{4}{|l|}{ Laboratory* } \\
\hline $\mathrm{pH}$ & $7.26(7.25-7.37)$ & $7.23(7.13-7.30)$ & .19 \\
\hline $\mathrm{PaO}_{2} / \mathrm{FiO}_{2}$ & $73.0(70.0-86.3)$ & $63.5(46.0-84.0)$ & .32 \\
\hline Arterial lactate, $\mathrm{mmol} / \mathrm{L}$ & $5.8(4.3-5.9)$ & $11.6(7.5-15.0)$ & .036 \\
\hline Platelet count, $\times 10^{3}$ & $59.0(38.0-90.0)$ & $37.0(22.0-88.0)$ & .16 \\
\hline Creatinine, mg/dL & $1.7(1.5-1.9)$ & $1.4(0.9-2.0)$ & .35 \\
\hline Total bilirubin, $\mathrm{mg} / \mathrm{dL}$ & $2.6(1.7-3.3)$ & $5.4(1.6-13.0)$ & .35 \\
\hline
\end{tabular}

$A R D S$, Acute respiratory distress syndrome; SOFA, sequential organ failure assessment score; $E C M O$, extracorporeal membrane oxygenation; $M A P$, mean arterial pressure; $C P R$, cardiopulmonary resuscitation; ECLS, extracorporeal life support. *Blood was sampled before starting ECLS.

difference was not statistically significant (18.0 [IQR, 13.0-20.0] vs 19.0 [IQR, 15.0-22.0], $P=.42$ ). Among the preprocedure laboratory parameters, arterial lactate was significantly higher in the nonsurvivor group (11.6 [IQR, 7.5-15.0] $\mathrm{mmol} / \mathrm{L}$ vs 5.8 [IQR, 4.3-5.9] mmol/L, $P=.036$ ), whereas platelet count was lower (37.0 [IQR, 22.0-88.0] vs 59.0 [IQR, 38.0-90.0], $P=.16$ ), and total bilirubin level was higher (5.4 [IQR, 1.6-13.0] vs 2.6 [IQR, 1.7-3.3] mg/dL, $P=.35$ ), both without statistical significance.

Data regarding infection sites and types of pathogen are presented in Table 2. The most common infection site was lung, accounting for $70 \%$ of cases. Thirty patients $(42.9 \%)$ gave positive blood cultures and they were all nonsurvivors. The most common pathogen was Acinetobacter baumannii, followed by Staphylococcus species and Pseudomonas aeruginosa. The distribution of pathogenic microorganisms tended to differ in the 2 groups, but the difference was not statistically significant probably because of the small number of survivors.

Table 3 shows the variables related to ECMO management. Intravenous corticosteroid was used in 18 patients $(25.4 \%)$. Lactate level 6 hours after the initiation of ECLS was significantly higher in the nonsurvivor group
(15.0 [IQR, 11.1-15.0] vs 5.2 [IQR, 4.7-5.4], $P=.002)$. Survivors received ECLS for longer than nonsurvivors (179.3 [IQR, 122.3-190.2] hours vs 26.4 [IQR, 12.7-56.9] hours, $P=.006)$.

\section{Clinical Outcomes of Venoarterial ECMO in Patients With Septic Shock and Comparison With Venoarterial ECMO in Patients With Cardiogenic Shock}

Of the total of 71 patients who underwent venoarterial ECMO for refractory septic shock, $11(15.5 \%)$ were successfully weaned off the ECMO after a median of 7.9 (IQR, 6.3-10.2) days (Figure 1). Of these patients, only 5 $(7.0 \%)$ survived to discharge (Figure 1). Finally, inhospital mortality was $93.0 \%$ (66 of 71) and 30-, 60-, and 90-day mortality rates were $87.3 \%, 91.5 \%$, and $93.0 \%$, respectively. A color change was noted in the affected limb of 1 patient whose distal limb had not been perfused via an antegrade perfusion catheter before the start of ECMO and the distal limb was perfused thereafter. No long-term disability was reported in the 5 hospital survivors.

For comparison, 253 patients who received venoarterial ECMO for cardiogenic shock during the same study period 
TABLE 2. Infection sites and types of pathogenic microorganisms

\begin{tabular}{llcc}
\hline \multicolumn{1}{c}{ Variable } & $\begin{array}{c}\text { Survivors } \\
(\mathbf{n}=\mathbf{5})\end{array}$ & $\begin{array}{c}\text { Nonsurvivors } \\
(\mathbf{n}=\mathbf{6 6})\end{array}$ & $\begin{array}{c}\boldsymbol{P} \\
\text { value }\end{array}$ \\
\hline Infection site, $\mathrm{n}(\%)$ & & & .35 \\
Lung & $3(60.0)$ & $46(69.7)$ & \\
Blood & $0(0)$ & $10(15.2)$ & \\
Abdomen & $1(20.0)$ & $7(10.6)$ & \\
Urinary tract & $1(20.0)$ & $2(3.0)$ & \\
Other* & $0(0)$ & $1(1.5)$ & \\
Positive blood culture, n (\%) & $0(0)$ & $30(45.5)$ & .069 \\
Microorganism types, n $(\%)$ & & & .055 \\
Gram-positive & & & \\
$\quad$ Staphylococcus species & $1(20.0)$ & $11(16.7)$ & \\
Enterococcus species & $0(0)$ & $2(3.0)$ & \\
Other gram-positive bacteria $\dagger$ & $0(0)$ & $1(1.5)$ & \\
Gram-negative & & & \\
Acinetobacter baumannii & $0(0)$ & $15(22.7)$ & \\
$\quad$ Pseudomonas aeruginosa & $0(0)$ & $9(13.6)$ & \\
Klebsiella pneumonia & $0(0)$ & $6(9.1)$ & \\
Escherichia coli & $0(0)$ & $6(9.1)$ & \\
Enterobacter species & $1(20.0)$ & $1(1.5)$ & \\
Other gram-negative bacteria $\ddagger$ & $0(0)$ & $4(6.1)$ & \\
Fungal & & & \\
Candida species & $1(20.0)$ & $7(10.6)$ & \\
Cryotococcus & $0(0)$ & $1(1.5)$ & \\
Aspergillus & $0(0)$ & $1(1.5)$ & \\
Other & & & \\
Mycobacterium & $2(40.0)$ & $2(3.0)$ & \\
\hline
\end{tabular}

*A patient with skin infection. $\dagger$ Other gram-positive bacteria included Corynebacterium. $\ddagger$ Other gram-negative bacteria included Achromobacter xylosoxidans, Rickettsia tsutsugamushi, and Stenotrophomonas maltophilia.

served as controls (Table E1). Of these, 115 (45.5\%) were successfully weaned from the ECMO, a significantly higher proportion than in the patients with septic shock (Table E2). Survival to hospital discharge in the non-septic shock patients was $28.9 \%$, which was also significantly higher than in those with septic shock (Table E2). As shown in Figure 2, the overall survival of the septic shock patients who received venoarterial ECMO was lower than that of the patients with cardiogenic shock who received ECMO $(P<.001)$.

\section{DISCUSSION}

The efficacy of venoarterial ECMO for refractory septic shock in neonates and children has been established by several studies. ${ }^{10-14}$ It was reported that $87 \%$ of neonates and $74 \%$ of children who underwent venoarterial ECMO for septic shock survived to discharge. ${ }^{11,14}$ Reflecting these high survival rates, the guidelines of the American College of Critical Care Medicine and the Extracorporeal Life Support Organization currently recommend venoarterial ECMO for pediatric septic shock refractory to conventional treatment. ${ }^{5,15}$ In contrast, the mortality rates of adult patients who underwent venoarterial ECMO
TABLE 3. Extracorporeal life support data

\begin{tabular}{|c|c|c|c|}
\hline Variable & $\begin{array}{l}\text { Survivors } \\
(\mathbf{n}=\mathbf{5})\end{array}$ & $\begin{array}{l}\text { Nonsurvivors } \\
\qquad(\mathrm{n}=66)\end{array}$ & $\begin{array}{c}P \\
\text { value }\end{array}$ \\
\hline $\begin{array}{l}\text { Use of nafamostat } \\
\text { mesylate, } n(\%)\end{array}$ & $0(0)$ & $7(10.6)$ & $>.99$ \\
\hline $\begin{array}{l}\text { Use of } \\
\text { corticosteroid, } n(\%)\end{array}$ & $1(20.0)$ & $17(25.8)$ & $>.99$ \\
\hline Hemofiltration, n (\%) & $1(20.0)$ & $6(9.1)$ & .99 \\
\hline $\begin{array}{l}\text { Duration of ECLS, } \\
\text { hours }\end{array}$ & $179.3(122.3-190.2)$ & $26.4(12.7-56.9)$ & .006 \\
\hline Lactate, $6 \mathrm{~h}, \mathrm{mmol} / \mathrm{L}$ & $5.2(4.7-5.4)$ & $15.0(11.1-15.0)$ & .002 \\
\hline
\end{tabular}

for refractory septic shock have been relatively high, and the reported outcomes have been inconsistent. Brechot et al analyzed 14 adult patients who underwent venoarterial ECMO for cardiovascular dysfunction during septic shock and reported a satisfactory in-hospital mortality of $29 \%{ }^{16}$ However, they excluded patients with conserved cardiac function from their study and this favorable outcome might not accurately represent the real-world situation because the hemodynamic presentations of septic shock vary. Older children and adults are usually in a hyperdynamic state with high cardiac output, ${ }^{17}$ whereas infants and younger children commonly have cardiovascular dysfunction. ${ }^{10-12}$ Moreover, it is not realistic to use venoarterial ECMO only in patients manifesting refractory septic shock combined with cardiovascular dysfunction because venoarterial ECMO is seen as a treatment of last resort. Indeed, Huang et al and Park et al reported high in-hospital mortalities in their studies concerning venoarterial ECMO for adult refractory septic shock. ${ }^{3,4}$ In the current study, the in-hospital mortality rate of the 71 septic shock patients who received venoarterial ECMO was $93 \%$, which is higher than in other studies. These poor results might be explained by the following factors. First, we did not exclude hyperdynamic septic shock patients, whose prognosis is expected to be much worse. Second, more than half of the patients ( 37 of $71 ; 52.1 \%$ ) had liver cirrhosis, including post-liver transplantation patients. Although liver cirrhosis could not be statistically confirmed as a risk factor for mortality because of the lack of parameters representing its severity, such a high proportion of comorbid liver cirrhosis would undoubtedly be associated with high mortality. The relatively high SOFA scores (mean $\pm \mathrm{SD}, 18.2 \pm 4.0$ ) might also be related to the poor outcomes of the current study.

Some authors have reported poorer outcomes of venoarterial ECMO for septic shock in patients 60 years or older and have suggested the use of central ECMO on the basis of pediatric experience. ${ }^{3}$ Theoretically, central ECMO would appear to be superior to peripheral ECMO; however, many physicians and surgeons hesitate to use it because of 


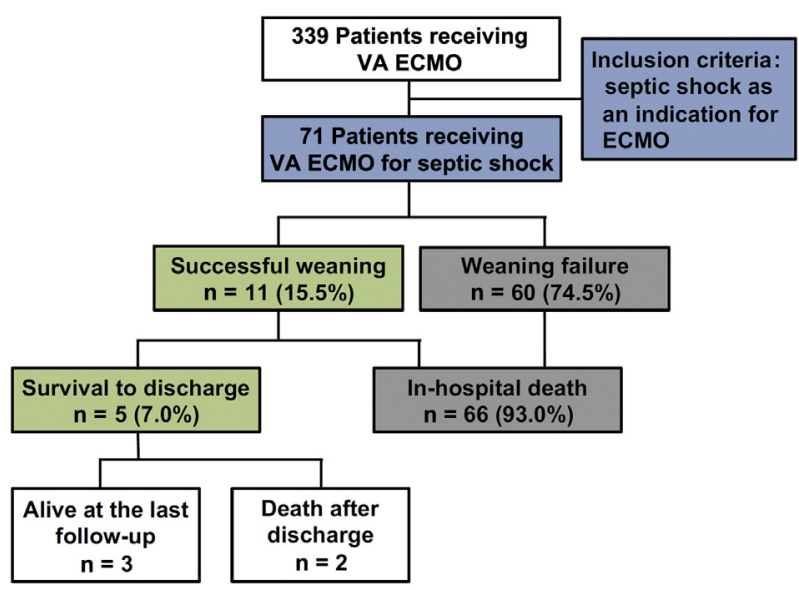

FIGURE 1. Flow chart of the outcomes of VA ECMO in septic shock patients. VA, Venoarterial; ECMO, extracorporeal membrane oxygenation.

its invasiveness and patients' comorbidities. In the real world, it is certainly challenging to switch cannulation routes from peripheral to central vessels via additional sternotomy or thoracotomy incisions especially in unstable patients. In the current study, peripheral cannulation was performed in all patients, as in the other studies. ${ }^{3,4,16}$

In this study, the arterial lactate level 6 hours after initiation of ECMO was the only significant factor associated with in-hospital mortality. A correlation between improved outcomes and early lactate clearance in severe sepsis and septic shock has been noted in other studies. ${ }^{18}$ In this context, early lactate clearance might also be an appropriate indicator of outcomes, or of the adequacy of the treatment, in patients who undergo venoarterial ECMO for septic shock. When the spectrum of patients' disease in our ECMO database was extended from septic shock to many other kinds of shock, the preprocedural arterial lactate level was found to be an independent predictor of the outcomes of

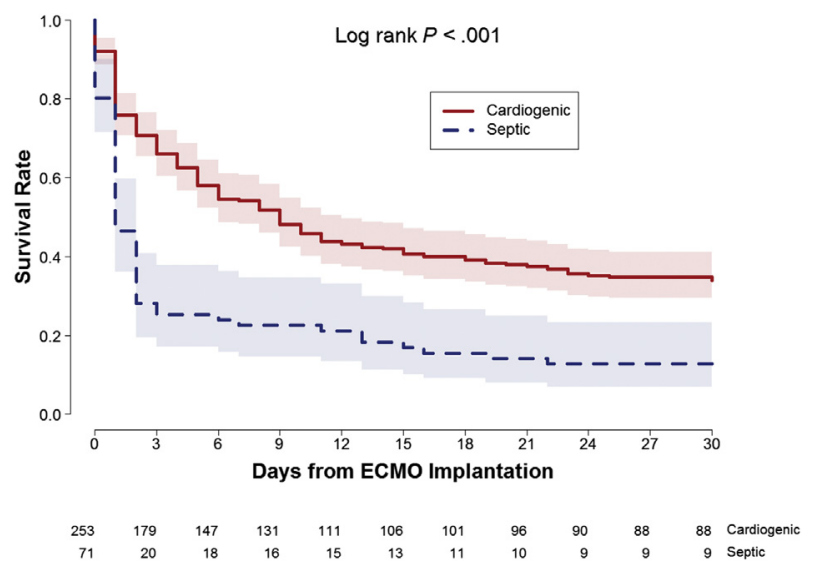

FIGURE 2. Overall survival of the septic shock patients receiving venoarterial extracorporeal membrane oxygenation $(E C M O)$ versus that of the cardiogenic shock patients receiving venoarterial ECMO. venoarterial ECMO. This suggests that the arterial lactate level could be helpful in deciding when to start ECLS.

Recently, gram-negative pathogens in intensive care unit patients have been causing alarm because of the increase in resistance to 3 or more classes of antibiotics. ${ }^{19}$ Gramnegative infections are often associated with inappropriate initial antibiotic therapy, which might eventually lead to multidrug resistance and increased hospital mortality. ${ }^{20,21}$ There was also a tendency in the present study for gramnegative pathogens to be more frequently detected in nonsurvivors than survivors $(20.0 \%$ vs $62.1 \% ; P=.055)$, although the statistical power was limited because of the small cohort size.

From the viewpoint of cost, \$6168 USD (\$4791 USD for ECMO setup, \$496 USD per day of ECMO maintenance) on average was charged only for the ECMO in this study cohort. Because approximately $80 \%$ of the cost can be covered by the national medical insurance system in this country, individual out-of-pocket payments might not be so high, but the social costs might be substantial. This might further raise the futility issue given such grave outcomes of ECMO in the setting of septic shock.

This study has some limitations. First, it was difficult to obtain high statistical power when comparing survivors and nonsurvivors because of the numerical imbalance between the 2 groups, although the study cohort was not small compared with other recent studies. Second, the absence of protocols or established guidelines for calling the ECMO team might have made it difficult to correctly time the onset of ECMO support. The clinical outcomes of venoarterial ECMO for septic shock in this study might have been poorer than in those performed in other clinical settings because of the generous policy of performing ECMO in many patients with advanced end-organ failure, such as liver transplant recipients.

\section{CONCLUSIONS}

The outcomes of venoarterial ECMO in refractory septic shock were poor in terms of weaning success and survival up to hospital discharge. Higher arterial lactate level preand post-ECMO might be associated with higher mortality risk. Further large-scale studies are needed to establish whether venoarterial ECMO has a role in the treatment of adult septic shock patients.

\section{Conflict of Interest Statement}

Authors have nothing to disclose with regard to commercial support.

\section{References}

1. Kennedy JH. The role of assisted circulation in cardiac resuscitation. JAMA. 1966;197:615-8.

2. Kurusz M, Zwischenberger JB. Percutaneous cardiopulmonary bypass for cardiac emergencies. Perfusion. 2002; 17:269-77. 
3. Huang CT, Tsai YJ, Tsai PR, Ko WJ. Extracorporeal membrane oxygenation resuscitation in adult patients with refractory septic shock. J Thorac Cardiovasc Surg. 2013;146:1041-6.

4. Park TK, Yang JH, Jeon K, Choi SH, Choi JH, Gwon HC, et al. Extracorporeal membrane oxygenation for refractory septic shock in adults. Eur J Cardiothorac Surg. 2015;47:e68-74.

5. ELSO. Extracorporeal Life Support Organization: ELSO guidelines. Available at: https://www.elso.org/Resources/Guidelines.aspx. Accessed September 24, 2017.

6. Dellinger RP, Levy MM, Carlet JM, Bion J, Parker MM, Jaeschke R, et al. Surviving Sepsis Campaign: international guidelines for management of severe sepsis and septic shock: 2008. Crit Care Med. 2008;36:296-327.

7. Park SJ, Kim JB, Jung SH, Choo SJ, Chung CH, Lee JW. Outcomes of extracorporeal life support for low cardiac output syndrome after major cardiac surgery. $J$ Thorac Cardiovasc Surg. 2014;147:283-9.

8. Lim JY, Kim JB, Choo SJ, Chung CH, Lee JW, Jung SH. Anticoagulation during extracorporeal membrane oxygenation; nafamostat mesilate versus heparin. Ann Thorac Surg. 2016;102:534-9.

9. Chen YS, Chao A, Yu HY, Ko WJ, Wu IH, Chen RJ, et al. Analysis and results of prolonged resuscitation in cardiac arrest patients rescued by extracorporeal membrane oxygenation. J Am Coll Cardiol. 2003;41:197-203.

10. Maclaren G, Butt W, Best D, Donath S, Taylor A. Extracorporeal membrane oxygenation for refractory septic shock in children: one institution's experience. Pediatr Crit Care Med. 2007:8:447-51.

11. MacLaren G, Butt W, Best D, Donath S. Central extracorporeal membrane oxygenation for refractory pediatric septic shock. Pediatr Crit Care Med. 2011;12:133-6.

12. UK collaborative randomised trial of neonatal extracorporeal membrane oxygenation. UK Collaborative ECMO Trial Group. Lancet. 1996;348:75-82.

13. McCune S, Short BL, Miller MK, Lotze A, Anderson KD. Extracorporeal membrane oxygenation therapy in neonates with septic shock. J Pediatr Surg. 1990; 25:479-82.
14. Hocker JR, Simpson PM, Rabalais GP, Stewart DL, Cook LN. Extracorporea membrane oxygenation and early-onset group B streptococcal sepsis. Pediatrics 1992;89:1-4.

15. Brierley J, Carcillo JA, Choong K, Cornell T, Decaen A, Deymann A, et al. Clinical practice parameters for hemodynamic support of pediatric and neonatal septic shock: 2007 update from the American College of Critical Care Medicine. Crit Care Med. 2009;37:666-88.

16. Brechot N, Luyt CE, Schmidt M, Leprince P, Trouillet JL, Leger P, et al. Venoarterial extracorporeal membrane oxygenation support for refractory cardiovascular dysfunction during severe bacterial septic shock. Crit Care Med. 2013;41: 1616-26.

17. Maclaren G, Butt W. Extracorporeal membrane oxygenation and sepsis. Crit Care Resusc. 2007;9:76-80.

18. Nguyen HB, Rivers EP, Knoblich BP, Jacobsen G, Muzzin A, Ressler JA, et al Early lactate clearance is associated with improved outcome in severe sepsis and septic shock. Crit Care Med. 2004;32:1637-42.

19. Zilberberg MD, Shorr AF. Prevalence of multidrug-resistant Pseudomonas aeruginosa and carbapenem-resistant Enterobacteriaceae among specimens from hospitalized patients with pneumonia and bloodstream infections in the United States from 2000 to 2009. J Hosp Med. 2013;8:559-63.

20. Iregui M, Ward S, Sherman G, Fraser VJ, Kollef MH. Clinical importance of delays in the initiation of appropriate antibiotic treatment for ventilator-associated pneumonia. Chest. 2002;122:262-8.

21. Zilberberg MD, Shorr AF, Micek ST, Vazquez-Guillamet C, Kollef MH. Multidrug resistance, inappropriate initial antibiotic therapy and mortality in gramnegative severe sepsis and septic shock: a retrospective cohort study. Crit Care. 2014;18:596.

Key Words: extracorporeal membrane oxygenation, septic shock, survival

Readers who found these articles interesting may also like to read the following papers found in recent and future issues of our sister publications, Seminars in Thoracic and Cardiovascular Surgery and Operative Techniques in Thoracic and Cardiovascular Surgery!

\section{Adult: Mechanical Circulatory Support}

STATE OF THE ART: Less Invasive Surgical Approaches for Left Ventricular Assist Device Implantation. Marcel Ricklefs. Semin Thoracic Surg 2018: 1-6

ORIGINAL SUBMISSION: Implantation of the HeartMate 3 -Description of the Surgical Technique. Friedhelm Beyersdorf. Oper Tech Thorac Cardiovasc 2018: In press

ORIGINAL SUBMISSION: Greater Omentum Wrapping to Treat Systemic Vad Infections. Sebastian Schulte Eistrup. Oper Tech Thorac Cardiovasc 2018: In press 
TABLE E1. Baseline characteristics of cardiogenic shock and septic shock groups

\begin{tabular}{|c|c|c|c|}
\hline Variable & Cardiogenic shock $(n=253)$ & Septic shock $(n=71)$ & $P$ value \\
\hline Age, y & $61.0(51.0-71.0)$ & $58.0(49.0-65.0)$ & .044 \\
\hline Male sex, n (\%) & $154(60.9)$ & $40(56.3)$ & .58 \\
\hline Body mass index, $\mathrm{kg} / \mathrm{m}^{2}$ & $23.4(21.2-25.8)$ & $22.1(19.6-25.7)$ & .063 \\
\hline Body surface area, $\mathrm{m}^{2}$ & $1.68(1.55-1.80)$ & $1.62(1.52-1.73)$ & .010 \\
\hline Diabetes mellitus, n (\%) & $58(22.9)$ & $19(26.8)$ & .61 \\
\hline Hypertension, $\mathrm{n}(\%)$ & $99(39.1)$ & $20(28.2)$ & .12 \\
\hline Chronic renal failure, n (\%) & $16(6.3)$ & $6(8.5)$ & .72 \\
\hline Liver cirrhosis, $\mathrm{n}(\%)$ & $19(7.5)$ & $37(52.1)$ & $<.001$ \\
\hline CPR before ECLS, n (\%) & $80(31.6)$ & $9(12.7)$ & .003 \\
\hline \multicolumn{4}{|l|}{ Laboratory* } \\
\hline $\mathrm{pH}$ & $7.29(7.18-7.37)$ & $7.24(7.15-7.30)$ & .013 \\
\hline Arterial lactate, $\mathrm{mmol} / \mathrm{L}$ & $9.2(4.8-13.7)$ & $11.1(6.3-15.0)$ & .041 \\
\hline Platelet count, $\times 10^{3}$ & $128.0(78.0-197.0)$ & $38.0(22.5-89.0)$ & $<.001$ \\
\hline Creatinine, mg/dL & $1.4(1.0-2.0)$ & $1.5(0.9-2.0)$ & .65 \\
\hline Total bilirubin, $\mathrm{mg} / \mathrm{dL}$ & $1.4(0.9-2.9)$ & $4.5(1.7-12.2)$ & $<.001$ \\
\hline Hemofiltration, $\mathrm{n}(\%)$ & $26(10.3)$ & $7(9.9)$ & $>.99$ \\
\hline Duration of ECLS, hours & $71.0(27.6-132.9)$ & $27.5(13.4-68.2)$ & .001 \\
\hline
\end{tabular}

CPR, Cardiopulmonary resuscitation; $E C L S$, extracorporeal life support. *Blood was sampled before starting ECLS.

TABLE E2. Clinical outcomes of cardiogenic shock and septic shock groups

\begin{tabular}{lccc}
\hline \multicolumn{1}{c}{ Outcomes } & Cardiogenic shock $(\mathbf{n}=\mathbf{2 5 3})$ & Septic shock $(\mathbf{n}=\mathbf{7 1})$ & $\boldsymbol{P}$ value \\
\hline Successful weaning, $\mathrm{n}(\%)$ & $115(45.5)$ & $11(15.5)$ & \\
Survival to discharge, $\mathrm{n}(\%)$ & $73(28.9)$ & $5(7.0)$ & $<.001$ \\
30-d mortality, $\mathrm{n}(\%)$ & $167(66.0)$ & $62(87.3)$ & $<.001$ \\
60-d mortality, $\mathrm{n}(\%)$ & $176(69.6)$ & $65(91.5)$ & .001 \\
90-d mortality, $\mathrm{n}(\%)$ & $177(70.0)$ & $66(93.0)$ & $<.001$ \\
\hline
\end{tabular}

